\title{
Cohortes de premedicación en endoscopia alta con simeticona, N-acetilcisteína, Hedera helix y validación de la escala visual
}

\section{Cohorts of premedication for endoscopy of the upper gastrointestinal tract with simethicone, $\mathrm{N}$-acetylcysteine, Hedera helix and visual scale validation}

Camilo Blanco, MD, MSc, ${ }^{* *}$ (1) María Isabel Barreto-Guevara, ${ }^{2}$ (D) Yenny Lucía Walteros-Gordillo, ${ }^{3}$ (D) Néstor Armando Pinzón-Segura, ${ }^{4}$ (b) María del Carmen Rivera-Velasco, ${ }^{5}$ (D) José Bareño, MD, MSc. 6 (D)

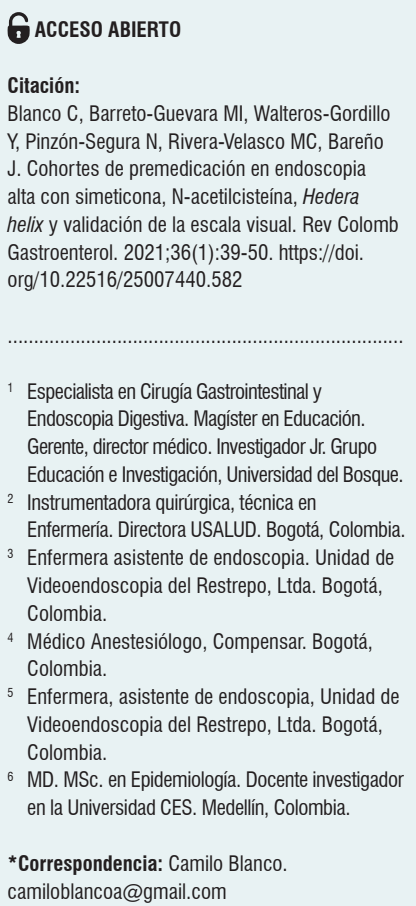

Especialista en Cirugía Gastrointestinal y Endoscopia Digestiva. Magíster en Educación. Gerente, director médico. Investigador Jr. Grupo Educación e Investigación, Universidad del Bosque. Instrumentadora quirúrgica, técnica en Enfermería. Directora USALUD. Bogotá, Colombia. Enfermera asistente de endoscopia. Unidad de Videoendoscopia del Restrepo, Ltda. Bogotá, Colombia.

${ }^{4}$ Médico Anestesiólogo, Compensar. Bogotá, Colombia.

5 Enfermera, asistente de endoscopia, Unidad de Videoendoscopia del Restrepo, Ltda. Bogotá, Colombia.

${ }^{6}$ MD. MSc. en Epidemiología. Docente investigador en la Universidad CES. Medellín, Colombia.

*Correspondencia: Camilo Blanco. camiloblancoa@gmail.com

Fecha recibido: $27 / 05 / 20$ Fecha aceptado: 28/10/20

\section{Resumen}

Los parámetros de calidad para endoscopia digestiva alta han introducido indicadores intraprocedimiento, dentro de los cuales la adecuada visibilidad de la mucosa, libre de saliva, moco o burbujas, puede aumentar la posibilidad de detección de lesiones en fase temprana. Sin embargo, el uso de mucolíticos y antiburbujas ha mostrado gran variabilidad de eficiencia según las soluciones, concentraciones, tiempos de exposición y escala de visibilidad aplicados. Objetivos: determinar la efectividad de diferentes soluciones de premedicación para la limpieza de la mucosa digestiva; validar, mediante una prueba de concordancia interobservador, una nueva escala de adecuada visualización de la mucosa (TVMS) para el esófago, estómago y duodeno; y reportar eventos adversos o complicaciones relacionadas con las soluciones utilizadas y los procedimientos realizados. Material y métodos: estudio de cohortes prospectivas comparativas. Se incluyeron 412 pacientes adultos, ASA I y ASA II, para endoscopia diagnóstica bajo sedación consciente, distribuidos en 6 cohortes similares, divididas en dos grupos: no premedicación, 2 cohortes $C 1$ (ayuno de 6 a 8 horas)y C2 (agua 100 $\mathrm{mL}$ ); premedicación, 4 cohortes C3 a C6 (C3: agua $100 \mathrm{~mL}+$ simeticona $1000 \mathrm{mg}$; C4: agua $100 \mathrm{~mL}+$ simeticona $200 \mathrm{mg}$ + N-acetilcisteína 600 mg; C5: agua $100 \mathrm{~mL}+$ simeticona $200 \mathrm{mg}+\mathrm{N}$-acetilcisteína $1000 \mathrm{mg}$; C6: agua $100 \mathrm{~mL}+$ simeticona $200 \mathrm{mg}+$ Hedera helix $70 \mathrm{mg}$ ). Se ingirió la solución 15 a 30 minutos antes del paso por cricofaríngeo. Se realizó la prueba de Kappa para medir la concordancia interobservador de la escala TVMS. Resultados: De 412 pacientes, 58 \% fueron de sexo femenino; 23 \% (136) fue de cohortes C1 y C2 y 67 \% (276) fue de cohortes C3 a C6. El tiempo medio de exposición a cada solución fue de 24,4 minutos. El volumen de lavado para lograr una adecuada visualización fue significativamente diferente entre ambos grupos: en los pacientes con premedicación se utilizaron $75,6 \mathrm{~mL}$, mientras que en los pacientes sin premedicación se utilizaron $124 \mathrm{~mL}(p=0,000)$, con una calidad de TVMS excelente de 88,7\% frente al 41,4\%, respectivamente. La cohorte $\mathrm{C} 4$ (agua $100 \mathrm{~mL}+$ simeticona $200 \mathrm{mg}+\mathrm{N}$-acetilcisteína $600 \mathrm{mg}$ ) mostró ser la más efectiva con una diferencia significativa $(p=0,001)$ frente a C1 (ayuno) y C2 (placebo con agua $100 \mathrm{~mL}$ ), y también tuvo una eficiencia superior frente a C3, C5 y C6 en su orden. No se presentaron eventos adversos o complicaciones en relación con la endoscopia, la sedación y los productos usados en la premedicación. Conclusiones: la solución más efectiva como premedicación para lograr una excelente visibilidad de la mucosa digestiva correspondió a la cohorte C4 (SIM $200+\mathrm{NAC} 600+\mathrm{H}_{2} \mathrm{O} 100 \mathrm{~mL}$ ). La escala TVMS propuesta es una herramienta muy completa y fácil de aplicar por más de un observador. La premedicación ingerida, con antiburbuja, mucolítico y agua hasta $100 \mathrm{~mL}$, entre 15 y 30 minutos previos a endoscopia, es segura en las condiciones descritas en este estudio.

Palabras clave

Premedicación, simeticona, N-acetilcisteína, esofagogastroduodenoscopia, TVMS, concordancia interobservador. 


\begin{abstract}
Quality parameters for upper gastrointestinal endoscopy have introduced intraprocedural indicators, including adequate mucosal visualization free of saliva, mucus, or bubbles, which may increase the possibility of earlystage injury detection. The use of mucolytics and anti-foaming agents has shown great efficiency variability depending on the type of solution, concentrations, exposure times and visibility scale applied. Objectives: To determine the effectiveness of different premedication solutions for cleaning the digestive mucosa; to validate, by means of an interobserver concordance test, a new scale for the adequate visualization of the mucosa (TVMS) for the esophagus, stomach, and duodenum; and to report adverse events or complications associated with the solutions used and the procedures performed. Material and methods: Prospective, comparative cohort study. 412 adult patients, ASA I and ASA II, were included for diagnostic endoscopy under conscious sedation. They were distributed in 6 similar cohorts and divided into two groups: non-premedication, 2 in $\mathrm{C} 1$ (fasting 6 to 8 hours) and C2 (water $100 \mathrm{~mL}$ ) cohorts; premedication, 4 C3 to C6 cohorts (C3: water $100 \mathrm{~mL}$ + simethicone $1000 \mathrm{mg}$; C4: water $100 \mathrm{ml}+$ simethicone $200 \mathrm{mg}+\mathrm{N}$-acetylcysteine $600 \mathrm{mg}$; C5: water 100 $\mathrm{ml}+$ simethicone $200 \mathrm{mg}+\mathrm{N}$-acetylcysteine $1000 \mathrm{mg}$; C6: water $100 \mathrm{ml}+$ simethicone $200 \mathrm{mg}+$ Hedera helix $70 \mathrm{mg}$ ). The solution was swallowed 15 to 30 minutes passing through the cricopharyngeus muscle. The Kappa test was performed to measure interobserver concordance of the TVMS scale. Results: Of 412 patients, $58 \%$ were female; $23 \%$ (136) were included in the C1 and C2 cohorts; and $67 \%$ (276) were in the $\mathrm{C} 3$ to $\mathrm{C} 6$ cohorts. The average exposure time to each solution was 24.4 minutes. The wash volume for proper visualization was significantly different between the two groups. In premedicated patients, $75.6 \mathrm{~mL}$ of solution were used, while in patients without premedication, $124 \mathrm{~mL}$ were used $(p=0.000)$, with an excellent quality of TVMS of $88.7 \%$ versus $41.4 \%$, respectively. The C4 cohort (water $100 \mathrm{~mL}+$ simethicone $200 \mathrm{mg}+$ $\mathrm{N}$-acetylcysteine $600 \mathrm{mg})$ was the most effective with a significant difference $(p=0.001)$ compared with the $\mathrm{C} 1$ (fasting) and C2 (placebo with water $100 \mathrm{~mL}$ ) cohorts. It also had better efficiency compared to the $\mathrm{C} 3, \mathrm{C} 5$ and C6 cohorts in that order. There were no adverse events or complications associated with endoscopy, sedation, or premedication products. Conclusions: The most effective solution as a premedication to achieve excellent visibility of the digestive mucosa was that used in the C4 cohort (SIM $200+\mathrm{NAC} 600+\mathrm{H}_{2} \mathrm{OR} 100 \mathrm{~mL}$ ). The proposed TVMS scale is a very complete and easy tool to apply by more than one observer. Premedication ingested, with anti-foam, mucolytic and water up to $100 \mathrm{~mL}$, between 15 and 30 minutes before endoscopy, is safe under the conditions described in this study.
\end{abstract}

\title{
Keywords
}

Premedication, Simethicone, N-acetylcysteine, Esophagogastroduodenoscopy, TVMS, Interobserver agreement.

\section{INTRODUCCIÓN}

La endoscopia digestiva alta actualmente tiene indicadores de calidad pre-, trans- y posprocedimiento (1-3). En el momento intraprocedimiento es fundamental mejorar la visibilidad de la mucosa digestiva alta, usualmente obstruida por saliva, moco, burbujas y líquido gástrico.

En 1964 Koga y Arakawa (4) en el tiempo preendoscópico, en pesquizaje de cáncer gástrico temprano mediante radiología con medio de contraste, eliminaron artefactos de visibilidad usando pronasa, una enzima mucolítica usada luego por Ida y colaboradores en 1991 para gastroendoscopia (5).

La efectividad de la pronasa (producto difícil de conseguir fuera de Japón, China y Corea) hizo que la premedicación se volviera una práctica rutinaria para la endoscopia digestiva alta, utilizando productos como $\mathrm{N}$-acetilcisteína (NAC), que es un mucolítico ampliamente accesible, solo o combinado con simeticona-dimeticona (SIM) (6-9), que es una sustancia antiespumante (10); aunque múltiples publicaciones no dan uniformidad en cuanto a las dosificaciones y resultados.

La variabilidad de reportes depende, en parte, de la forma de medir la visibilidad de la mucosa. En 2 metaanálisis (11, 12) se aprecia una gran disparidad en las escalas de visibilidad aplicadas, en ocasiones como la tasa de visibilidad mucosa (TVS; solo de la mucosa de segmentos del estómago) $(6,13,14)$ o como la escala total de visibilidad de la mucosa (TVMS; que incluye al esófago o duodeno) (15). Las escalas tienen una especial dificultad en su expresión cuantitativa, lo que genera confusión de las cifras difíciles de recordar; por esta razón, han surgido propuestas de pasar esas cifras a escalas cualitativas fáciles de recordar y aplicar (excelente, adecuado, inadecuado) (7).

La premedicación para mejorar la calidad de la visibilidad busca detectar lesiones en la fase temprana del esófago, duodeno y estómago, lo que es de especial interés para nuestro medio en la búsqueda de cáncer gástrico temprano o incipiente, debido a que es el séptimo cáncer más común 
en el mundo (16): en 2018 tuvo una incidencia global de 1033701 casos, mientras que en Colombia fue la primera causa de muerte por cáncer en hombres y la cuarta en mujeres (17). Su detección e intervención en la fase temprana ha modificado la supervivencia en países orientales a más del $90 \%$, frente a solo $10 \%$ a $20 \%$ a 5 años para cáncer gástrico avanzado (18-20).

Se usó por varios años la SIM líquida como el único producto de premedicación, pero los resultados internacionales alentadores, aunque discordantes al combinar SIM con NAC (incluidas dos publicaciones colombianas recientes) $(21,22)$, incentivaron los objetivos del presente estudio: comparar para determinar la mejor efectividad de diferentes soluciones de premedicación, una de las cuales incluyó un mucolítico no estudiado (Hedera hélix $[\mathrm{HH}]$ ); validar una nueva escala adaptada de visualización de esófago, estómago y duodeno; y reportar eventos adversos y complicaciones relacionadas con las soluciones y procedimientos de la endoscopia y la sedación consciente empleados.

\section{MATERIAL Y MÉTODOS}

\section{Selección de pacientes}

El estudio consiste en 6 cohortes prospectivas, comparativas en un centro ambulatorio de segundo nivel de Bogotá, realizado del 1 de mayo al 31 de julio de 2019 en pacientes de 18 años o más, informados sobre el objetivo del estudio y quienes dieron el consentimiento informado de su participación; se llevaron a endoscopia digestiva alta diagnóstica, con sospechas clínicas principales de dispepsia, enfermedad por reflujo gastroesofágico, disfagia no estudiada, malignidad o pesquizaje de cáncer gástrico o esofágico; y se clasificaron como ASA I y II, de acuerdo con la Sociedad Americana de Anestesiología (ASA).

Los criterios de exclusión fueron las lesiones tumorales o no tumorales generadoras de estenosis infranqueables o síndrome pilórico, endoscopia terapéutica, sangrado digestivo activo o reciente, embarazo, gastroparesia y reacciones alérgicas conocidas a medicamentos de la premedicación o de sedación.

El protocolo se realizó de acuerdo con la declaración de Helsinki y fue aprobada por el comité de ética de la unidad, en especial en dos aspectos: luego de la calificación final de la premedicación, todas las preparaciones que no fueran excelentes debían ser llevadas a ese nivel mediante la limpieza de la mucosa utilizando una solución de agua con SIM al 0,1 \% y en el volumen requerido para lograr un excelente aclaramiento mucoso; de este modo, se le aseguraba a todos los participantes total equidad en la posibilidad de detectar lesiones tempranas o avanzadas; y después de revisada la seguridad de una sola y pequeña dosis de $\mathrm{HH}$ y ante los efectos benéficos vistos en la limpieza de la mucosa digestiva en algunos pacientes no controlados que la habían ingerido para cuadros pulmonares, se decidió aprobar la cohorte 6 en la que se combina HH con SIM, cuyo resultado será planteado como recomendación de uso fuera de indicación (off-label).

\section{Diseño del estudio}

El tamaño de la muestra fue de 412 pacientes en 6 cohortes (Figura 1) (tamaño de la muestra mínimo de 355 para un nivel de confianza del $97 \%$ y error máximo admitido de $5 \%$, según la población de 1440 pacientes atendidos en los 2 años previos).

Todos los pacientes tuvieron una aleatorización simple (por una enfermera coordinadora) y ayuno mínimo de 8 horas. La enfermera coordinadora supervisó que la ingesta de cada solución se realizara entre 15 y 30 minutos antes del paso por el cricofaríngeo. Con la administración de anestésico local orofaríngeo, todos los procedimientos se realizaron bajo la sedación balanceada con propofol $(8 \mathrm{mg} / \mathrm{kg})$ y remifentanilo $(4 \mu \mathrm{g} / \mathrm{kg})$, en pacientes de ASA I y II; y se observaron como eventos adversos principales la reacción alérgica a los medicamentos, la depresión respiratoria que requiriera asistencia con presión positiva y broncoaspiración, de acuerdo con el protocolo institucional publicado (23).

Los pacientes se dividieron en dos grupos: no exposición (no premedicación) y exposición (premedicación). Al grupo de no exposición se le asignó 2 cohortes (C): C1: no solución (NS) y C2: agua $\left(\mathrm{H}_{2} \mathrm{O}\right) 100 \mathrm{~mL}$. Al grupo exposición se le asignó 4 cohortes $(\mathrm{C})$ : $\mathrm{C} 3$ : agua $100 \mathrm{~mL}+$ simeticona $1000 \mathrm{mg}\left(\mathrm{H}_{2} \mathrm{O}+\mathrm{SIM} 1000\right)$; C4: agua $100 \mathrm{~mL}+$ simeticona $200 \mathrm{mg}+\mathrm{N}$-acetilcisteína $600 \mathrm{mg}\left(\mathrm{H}_{2} \mathrm{O}+\mathrm{SIM}\right.$ $200+$ NAC 600); C5: agua $100 \mathrm{~mL}+$ simeticona $200 \mathrm{mg}+$ $\mathrm{N}$-acetilcisteína 1000 mg ( $\mathrm{H}_{2} \mathrm{O}+$ SIM 200 + NAC 1000); y C6: agua $100 \mathrm{~mL}+$ simeticona (SIM) $200 \mathrm{mg}+$ Hedera helix $(\mathrm{HH}) 70 \mathrm{mg}\left(\mathrm{H}_{2} \mathrm{O}+\mathrm{SIM} 200+\mathrm{HH} 70\right)$, con similitud en el número de pacientes por cohorte (Figura 1).

\section{Escala de visibilidad de la mucosa}

Se adoptó una escala mixta (cualitativa y cuantitativa) de visibilidad total de la mucosa (TVMS), modificada del sistema cualitativo de Elvas (7), proveniente del sistema cuantitativo de McNally (24). Se incluyeron niveles de valor de 1 a 4 según la limpieza de la mucosa, para 7 sitios del tracto digestivo alto ( 1 de esófago, 4 de estómago y 2 de duodeno), cuyas sumas totales (entre 7 y hasta 28 ) se llevaron a tres niveles cualitativos (excelente, adecuado e inadecuado), con cierta similitud, pero clara diferencia frente a otras escalas publicadas (Tabla 1) (15, 25-30). 


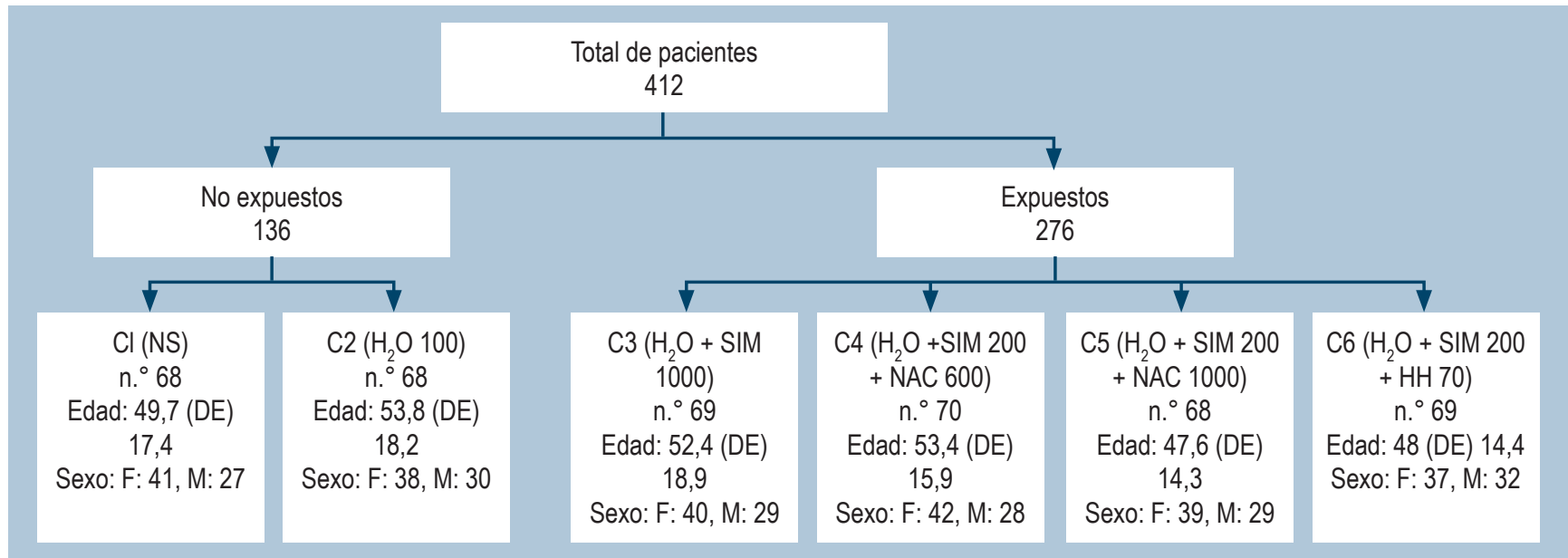

Figura 1. Características sociodemográficas de 6 cohortes. DE: desviación estándar; HH: Hedera helix; $\mathrm{H}_{2} \mathrm{O}$ : agua; NAC: N-acetilcisteína; NS: no solución; SIM: simeticona.

Tabla 1. Comparación entre las diferentes escalas de visibilidad de la mucosa (7)

\section{$\begin{array}{lll}\text { Escala y referencia Sitios evaluados } & \text { Calificación }\end{array}$}

\begin{tabular}{|c|c|c|c|c|}
\hline \multirow[t]{2}{*}{$\begin{array}{l}\text { Escala cuantitativa y } \\
\text { cualitativa, Blanco y } \\
\text { colaboradores (este } \\
\text { artículo) }\end{array}$} & $\begin{array}{l}7 \text { sitios: esófago, } 4 \text { sitios } \\
\text { gástricos (fondo, cuerpo } \\
\text { proximal, cuerpo distal y } \\
\text { antro), bulbo duodenal y D2. }\end{array}$ & $\begin{array}{l}\text { Excelente: } 7 \text { a } 14 \text { puntos. } \\
\text { Mucosa completamente } \\
\text { limpia o máximo requiere } \\
\text { solo aspiración. }\end{array}$ & $\begin{array}{l}\text { Adecuado: } 15 \text { a } 21 \text { puntos. } \\
\text { Requiere lavar con menos de } \\
50 \text { mL en máximo } 3 \text { sitios. }\end{array}$ & $\begin{array}{l}\text { Inadecuado: } 22 \text { a } 28 \text { puntos. } \\
\text { Requiere lavar con más de } \\
50 \text { mL en } 3 \text { o más sitios. }\end{array}$ \\
\hline & $\begin{array}{l}4 \text { puntajes por sitio: } \\
\text { 1. Sin moco ni burbujas. }\end{array}$ & $\begin{array}{l}\text { 2. Con moco flotante } 0 \\
\text { no adherente o burbujas } \\
\text { escasas, succionables, que } \\
\text { no obstruyen la visión. }\end{array}$ & $\begin{array}{l}\text { 3. Moco adherente o burbujas } \\
\text { abundantes, no succionables, } \\
\text { que obstruyen la visión y } \\
\text { requieren menos de } 50 \mathrm{~mL} \text { de } \\
\text { agua para limpiar. }\end{array}$ & $\begin{array}{l}\text { 4. Moco adherente } 0 \\
\text { burbujas abundantes, no } \\
\text { succionables, que obstruyen } \\
\text { la visión y requieren más de } \\
50 \mathrm{~mL} \text { de agua para limpiar. }\end{array}$ \\
\hline Elvas y colaboradores (7) & $\begin{array}{l}3 \text { sitios: esófago, estómago } \\
\text { y duodeno. }\end{array}$ & $\begin{array}{l}\text { Excelente moco no } \\
\text { adherente y visión clara de } \\
\text { la mucosa (incluida en el } \\
\text { uso de aspiración). }\end{array}$ & $\begin{array}{l}\text { Adecuado moco adherido } \\
\text { que obstruye la visión de la } \\
\text { mucosa y requiere lavado con } \\
\text { agua. }\end{array}$ & $\begin{array}{l}\text { Inadecuado moco grueso } \\
\text { o residuo alimentario no } \\
\text { susceptible de aspiración. }\end{array}$ \\
\hline $\begin{array}{l}\text { Escala A: Bhandari y } \\
\text { colaboradores (25), Lee y } \\
\text { colaboradores (26), Chang } \\
\text { y colaboradores (15) }\end{array}$ & $\begin{array}{l}7 \text { sitios: esófago, } 4 \text { sitios } \\
\text { gástricos (fondo, cuerpo } \\
\text { proximal, cuerpo distal y } \\
\text { antro), bulbo duodenal y D2. }\end{array}$ & $\begin{array}{l}\text { Puntaje 1: sin moco } \\
\text { adherente y visión clara de } \\
\text { la mucosa. }\end{array}$ & $\begin{array}{l}\text { Puntaje 2: fino recubrimiento } \\
\text { del moco, sin obstruir la } \\
\text { visión. }\end{array}$ & $\begin{array}{l}\text { Puntaje 3: moco adherente } \\
\text { que obstruye la visión. }\end{array}$ \\
\hline $\begin{array}{l}\text { Escala B: Bertoni y } \\
\text { colaboradores ( } 27) \text {, } \\
\text { McNally y colaboradores } \\
\text { (24) }\end{array}$ & $\begin{array}{l}6 \text { sitios: esófago, fondo, } \\
\text { cuerpo, incisura, antro y } \\
\text { duodeno (o yeyuno si hay } \\
\text { gastroyeyunostomía). }\end{array}$ & $\begin{array}{l}\text { Puntaje } 1 \text { o 2: } \sin \text { o con } \\
\text { mínima cantidad de espuma } \\
\text { y burbujas. }\end{array}$ & $\begin{array}{l}\text { Puntaje 3: cantidad moderada } \\
\text { de espuma o burbujas. }\end{array}$ & $\begin{array}{l}\text { Puntaje 4: cantidad } \\
\text { abundante que oscurece } \\
\text { la superficie mucosa } \\
\text { (necesidad de lavado). }\end{array}$ \\
\hline $\begin{array}{l}\text { Escala C: Kuo y } \\
\text { colaboradores (28), Asl y } \\
\text { colaboradores (29), Chang } \\
\text { y colaboradores (30) }\end{array}$ & $\begin{array}{l}4 \text { sitios gástricos: fondo, } \\
\text { cuerpo proximal, cuerpo } \\
\text { distal y antro. }\end{array}$ & $\begin{array}{l}\text { Puntaje } 1 \text { o 2: moco no } \\
\text { adherente o pequeña } \\
\text { cantidad, que no obstruye } \\
\text { la visión. }\end{array}$ & $\begin{array}{l}\text { Puntaje 3: gran cantidad de } \\
\text { moco con menos de } 50 \mathrm{~mL} \text { de } \\
\text { agua para el aclaramiento. }\end{array}$ & $\begin{array}{l}\text { Puntaje 4: gran cantidad de } \\
\text { moco con más de } 50 \mathrm{~mL} \text { de } \\
\text { agua para el aclaramiento. }\end{array}$ \\
\hline
\end{tabular}

*Adaptado de: Elvas L et al. Endoscopy. 2017;49(2):139-145. 
Se realizó el lavado de la mucosa con agua y simeticona a muy baja disolución $(0,1 \%)$ solo al haber completado la visualización de los 7 sitios, aunque lavar antes podía modificar el valor dada la continuidad anatómica de los segmentos y de los tres órganos (Figura 2).

Los rangos de valor fueron de 1 a 4 :

1. sin moco ni burbujas;

2. con moco flotante o no adherente o burbujas escasas, succionables, que no obstruyen la visión;
3. con moco adherente o burbujas abundantes, no succionables, que obstruyen la visión y requieren menos de $50 \mathrm{~mL}$ de agua para limpiar;

4. con moco adherente o burbujas abundantes, no succionables, que obstruyen la visión y requieren más de 50 $\mathrm{mL}$ de agua para limpiar.

El rango total de puntaje va entre 7 y 28 puntos, que se agruparon en tres grupos cualitativos: preparación excelente ( 7
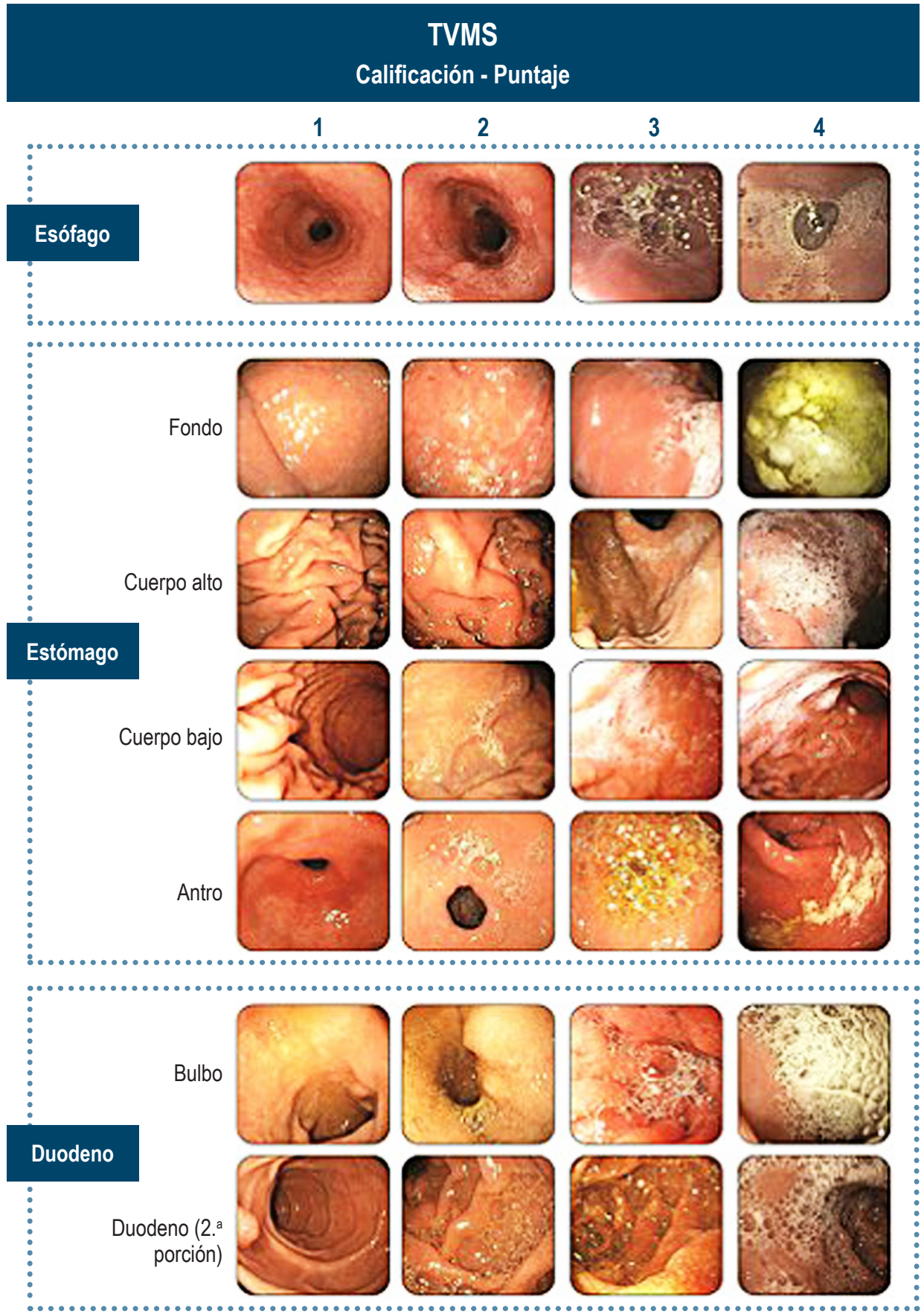

Figura 2. Escala de visibilidad total de la mucosa digestiva alta (TVMS). 
a 14 puntos), preparación adecuada ( 15 a 21 puntos) y preparación inadecuada ( 22 a 28 puntos).

La TVMS se evaluó simultáneamente por CB (endoscopista, más de 120000 endoscopias) y YW (enfermera, más de 32000 endoscopias), en dos formatos iguales, privados e independientes, entregados inmediatamente terminada la endoscopia a la enfermera coordinadora. Se cuantificó el volumen necesario de lavado intraendoscopia. Dada la variabilidad en el tiempo de toma de biopsias y en la valoración de lesiones de alto riesgo o malignas, se decidió no incluir el tiempo total de la endoscopia (31).

\section{Análisis estadístico}

Se realizó un análisis univariado que se presentó de acuerdo con la naturaleza de la variable. Las pruebas de hipótesis por subgrupos de exposición se contrastaron con la prueba de Kruskall Wallis, debido al incumplimiento de la normalidad de las variables analizadas. Se llevó a cabo un análisis multivariado exploratorio con el fin de conocer el efecto de las exposiciones sobre el puntaje de visibilidad de la mucosa digestiva, a través de un modelo de regresión lineal simple; se dejaron como variables predictivas la edad, el sexo, el volumen de lavado y el tiempo de premedicación. Las variables fueron significativas en el modelo cuando el valor de $p$ fue $<0,05$ y se buscó el modelo más parsimonioso. Además, se realizó un análisis de concordancia entre los dos evaluadores de la TVMS a partir de la prueba de independencia chi cuadrado $\left(\chi^{2}\right)$, una prueba de hipótesis de proporción de una muestra para un acuerdo superior a $70 \%$ para el puntaje de visibilidad de la mucosa; y posteriormente se estimó el índice kappa para la variable categórica (excelente, adecuada, inadecuada) (1). Los datos fueron procesados en un software R versión 3.2.0.

\section{RESULTADOS}

Se incluyeron 412 pacientes con un promedio de edad de 51 años (DE: 17), el $58 \%(\mathrm{n}=237)$ de sexo femenino. El $23 \%(\mathrm{n}=136)$ quedó asignado a las cohortes 1 y 2 de no exposición (no premedicación) y el $67 \%(\mathrm{n}=276)$, a las cohortes 3 a 6, de exposición (premedicación). El tiempo promedio de exposición (período entre la ingesta de la solución y el paso por el cricofaríngeo) fue de 24,3 minutos (DE: 4,4). En estas distribuciones no hay diferencias significativas según el valor de $p$. A su vez, el volumen del lavado requerido para lograr una excelente visibilidad muestra una diferencia significativa en las cohortes de premedicación (promedio de 75,6 mL; DE: 51,5) frente a las de no premedicación (promedio de 124,9 mL; DE: 76,5) $(p=0,000)$ (Tabla 2).
Tabla 2. Características sociodemográficas de la población de estudio

\begin{tabular}{|c|c|c|c|}
\hline \multirow[t]{2}{*}{ Características } & \multicolumn{2}{|c|}{ Premedicación } & \multirow{2}{*}{$\begin{array}{l}\text { Valo } \\
\text { de } p\end{array}$} \\
\hline & $\begin{array}{c}\text { No }(n=136) \\
(23 \%)\end{array}$ & $\begin{array}{l}\text { Si }(n=276) \\
(67 \%)\end{array}$ & \\
\hline Sexo (femenino, masculino) & $\begin{array}{l}79(33,3), \\
57(32,6)\end{array}$ & $\begin{array}{c}158(66,7), \\
118(67,4)\end{array}$ & 0,871 \\
\hline Edad promedio (DE) & $52,1(17,9)$ & $50,2(16,1)$ & 0,304 \\
\hline $\begin{array}{l}\text { Tiempo total de } \\
\text { premedicación }(n=345) \\
\text { media (DE) } \min \end{array}$ & $24,4(4,3)$ & $24,2(4,4)$ & 0,683 \\
\hline $\begin{array}{l}\text { Volumen de lavado }(n=215) \\
\text { media }(D E)\end{array}$ & $124,9(76,5)$ & $75,6(51,5)$ & 0,000 \\
\hline
\end{tabular}

A su vez, en las 2 cohortes de no premedicación ( $\mathrm{C} 1$ y $\mathrm{C} 2$ ), la media del volumen de lavado fue entre 126 y 123 $\mathrm{mL}$ para el $82 \%$ al $87 \%$ de los pacientes de cada cohorte (intervalo de confianza [IC] del 95\%); mientras que en las de premedicación (C3, C4, C5 y C6), la media estuvo entre 59 y $84 \mathrm{~mL}$, para el $32 \%$ y el $43 \%$ (IC $95 \%$ ), y el grupo C4 $\left(\mathrm{H}_{2} \mathrm{O}+\mathrm{SIM} 200+\mathrm{NAC} 600\right)$ fue el que requirió menos volumen (Figura 3).

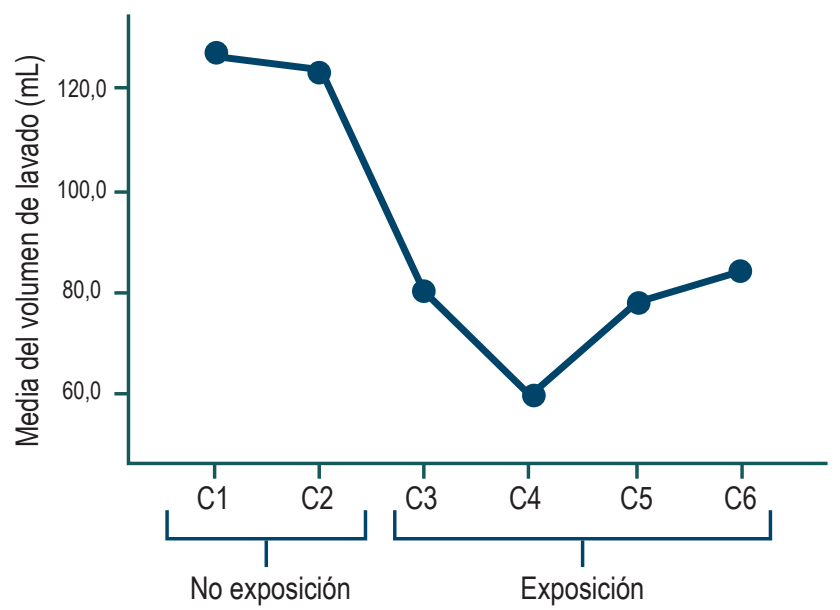

Figura 3. Asociación entre premedicación y lavado intraprocedimiento ( $\mathrm{mL}$ de agua con simeticona al $0,1 \%$ ).

$\mathrm{Al}$ realizar el comparativo cohorte a cohorte, se encuentran diferencias significativas entre $\mathrm{C} 1$ (NS) y las cohortes de premedicación $\mathrm{C} 4$ y C5, esto demuestra que, con el uso de estas dos soluciones, la visibilidad de la mucosa es mejor cuando se requiere menos volumen de agua adicional (Tabla 3).

Al realizar la conversión del puntaje cuantitativo de la TVMS a la clasificación cualitativa, incluidas todas las 
cohortes, se obtuvo un porcentaje de visibilidad excelente en el $72 \%$, adecuada en el $26 \%$ e inadecuada en el $2 \%$, para la totalidad de los pacientes. Este resultado obligó a descartar a la visibilidad inadecuada para análisis subsecuentes, pues su recuento mínimo esperado era de 33,67 pacientes (de acuerdo con las pruebas de $\chi^{2}$ de Pearson) (Tabla 4).

Tabla 3. Comparaciones múltiples entre premedicaciones y volumen de lavado

\begin{tabular}{|c|c|c|c|c|}
\hline Cohorte (I) & $\begin{array}{c}\text { Cohorte } \\
\text { (J) }\end{array}$ & $\begin{array}{c}\text { Diferencia de } \\
\text { medias (I-J) }\end{array}$ & $\begin{array}{l}\text { Valor } \\
\text { de } p\end{array}$ & IC $95 \%$ \\
\hline C1 (NS) & $\begin{array}{l}\mathrm{C} 2 \\
\mathrm{C} 3 \\
\mathrm{C} 4 \\
\mathrm{C} 5 \\
\mathrm{C} 6\end{array}$ & $\begin{array}{c}2,70 \\
45,95 \\
66,35 \\
48,44 \\
42,84\end{array}$ & $\begin{array}{l}1,000 \\
0,066 \\
0,001^{*} \\
0,039^{*} \\
0,050^{*}\end{array}$ & $\begin{array}{c}-32,917: 38,317 \\
-1,737: 93,643 \\
20,793: 111,910 \\
1,516: 95,374 \\
, 029: 85,647\end{array}$ \\
\hline $\mathrm{C} 2\left(\mathrm{H}_{2} \mathrm{O}\right)$ & $\begin{array}{l}\text { C1 } \\
\text { C3 } \\
\text { C4 } \\
\text { C5 } \\
\text { C6 }\end{array}$ & $\begin{array}{l}-2,70 \\
43,25 \\
63,65 \\
45,74 \\
40,14\end{array}$ & $\begin{array}{l}1,000 \\
0,104 \\
0,001^{*} \\
0,064 \\
0,085\end{array}$ & $\begin{array}{c}-38,317: 32,917 \\
-4,783: 91,289 \\
17,731: 109,572 \\
-1,535: 93,026 \\
-3,056: 83,332\end{array}$ \\
\hline $\begin{array}{l}\mathrm{C} 3\left(\mathrm{H}_{2} \mathrm{O}+\mathrm{SIM}\right. \\
1000)\end{array}$ & $\begin{array}{l}\mathrm{C} 1 \\
\mathrm{C} 2 \\
\mathrm{C} 4 \\
\mathrm{C} 5 \\
\mathrm{C} 6\end{array}$ & $\begin{array}{c}-45,95 \\
-43,25 \\
20,40 \\
2,49 \\
-3,11\end{array}$ & $\begin{array}{l}0,066 \\
0,104 \\
0,900 \\
1,000 \\
1,000\end{array}$ & $\begin{array}{l}-93,643: 1,737 \\
-91,289: 4,783 \\
-35,409: 76,206 \\
-54,440: 59,424 \\
-56,702: 50,471\end{array}$ \\
\hline $\begin{array}{l}\mathrm{C} 4\left(\mathrm{H}_{2} \mathrm{O}+\mathrm{SIM}\right. \\
200+\mathrm{NAC} \\
600)\end{array}$ & $\begin{array}{l}\mathrm{C} 1 \\
\mathrm{C} 2 \\
\mathrm{C} 3 \\
\mathrm{C} 5 \\
\mathrm{C} 6\end{array}$ & $\begin{array}{l}-66,35 \\
-63,65 \\
-20,40 \\
-17,91 \\
-23,51\end{array}$ & $\begin{array}{l}0,001^{*} \\
0,001^{*} \\
0,900 \\
0,937 \\
0,780\end{array}$ & $\begin{array}{c}-111,910:-20,793 \\
-109,572:-17,731 \\
-76,206: 35,409 \\
-73,065: 37,252 \\
-75,212: 28,185\end{array}$ \\
\hline $\begin{array}{l}\mathrm{C} 5\left(\mathrm{H}_{2} \mathrm{O}+\mathrm{SIM}\right. \\
200+\mathrm{NAC} \\
1000)\end{array}$ & $\begin{array}{l}\mathrm{C} 1 \\
\mathrm{C} 2 \\
\mathrm{C} 3 \\
\mathrm{C} 4 \\
\mathrm{C} 6\end{array}$ & $\begin{array}{c}-48,44 \\
-45,74 \\
-2,49 \\
17,91 \\
-5,61\end{array}$ & $\begin{array}{l}0,039^{*} \\
0,064 \\
1,000 \\
0,937 \\
1,000\end{array}$ & $\begin{array}{l}-95,374:-1,516 \\
-93,026: 1,535 \\
-59,424: 54,440 \\
-37,252: 73,065 \\
-58,517: 47,303\end{array}$ \\
\hline $\begin{array}{l}\mathrm{C} 6\left(\mathrm{H}_{2} \mathrm{O}+\mathrm{SIM}\right. \\
200+\mathrm{HH} 70)\end{array}$ & $\begin{array}{l}\mathrm{C} 1 \\
\mathrm{C} 2 \\
\mathrm{C} 3 \\
\mathrm{C} 4 \\
\mathrm{C} 5\end{array}$ & $\begin{array}{c}-42,84 \\
-40,14 \\
3,11 \\
23,51 \\
5,61\end{array}$ & $\begin{array}{l}0,050 \\
0,085 \\
1,000 \\
0,780 \\
1,000\end{array}$ & $\begin{array}{l}-85,647:-0,029 \\
-83,332: 3,056 \\
-50,471: 56,702 \\
-28,185: 75,212 \\
-47,303: 58,517\end{array}$ \\
\hline
\end{tabular}

*Prueba HSD de Tukey. La diferencia de medias es significativa en el nivel 0,05. HSD: honestly-significant-difference.

Tabla 4. Distribución de calidad TVMS (global TVMS)

\begin{tabular}{|lc|}
\hline \multicolumn{1}{|c|}{ Distribución cualitativa de visibilidad } & $\mathbf{n}=\mathbf{4 1 2}(\%)$ \\
\hline Excelente $(7$ a 14) & $297(72,1)$ \\
\hline Adecuado (15 a 21$)$ & $106(25,7)$ \\
\hline Inadecuado (22 a 28$)$ & $9(2,2)$ \\
\hline
\end{tabular}

Hecha la anterior precisión, se evidencia que para las cohortes de premedicación (C3 a C6) se logró el 88,7 \% de visibilidad excelente frente al 41,4\% de las dos cohortes de no premedicación (Tabla 5 ).

Tabla 5. Distribución de cohortes de premedicación y no premedicación frente a la calidad de visibilidad de la mucosa según la TVMS

\begin{tabular}{llcr}
\hline \multicolumn{1}{c}{ Cohortes } & \multicolumn{2}{c}{ Calidad de visibilidad de TVMS } & Total (\%) \\
\cline { 2 - 3 } & $\begin{array}{l}\text { Excelente } \\
(\mathbf{7} \text { a 14) }\end{array}$ & $\begin{array}{c}\text { Adecuada } \\
\text { (15 a 21) }\end{array}$ & \\
\cline { 2 - 3 } & $\mathbf{n ( \% )}$ & $\mathbf{n}(\%)$ & \\
\hline $\begin{array}{l}\text { Premedicación (C3, } \\
\text { C4, C5, C6) }\end{array}$ & $244(88,7)$ & $31(11,3)$ & $128(100)$ \\
$\begin{array}{l}\text { No premedicación (C1, } \\
\text { C2) }\end{array}$ & $53(41,4)$ & $75(58,6)$ & \\
\hline Total & $297(73,7)$ & $106(26,3)$ & $403(100)$
\end{tabular}

A su vez, al realizar el comparativo de calidad cualitativa de visibilidad cohorte a cohorte, se encontró la cohorte $\mathrm{C} 4$ $\left(\mathrm{H}_{2} \mathrm{O}+\mathrm{SIM} 200+\right.$ NAC 600) asociada con la mejor visibilidad (excelente en el 92,9\%). La segunda mejor cohorte fue $\mathrm{C} 3\left(\mathrm{H}_{2} \mathrm{O}+\mathrm{SIM} 1000 ; 89,9 \%\right)$, seguida de $\mathrm{C} 5\left(\mathrm{H}_{2} \mathrm{O}+\right.$ SIM 200 + NAC 1000; 88,1\%) y, finalmente, C6 ( $\mathrm{H}_{2} \mathrm{O} 100$ $\mathrm{mL}+$ SIM $200 \mathrm{mg}$ + HH 70 mg; con 84,1\%); esta última es menos eficiente que las otras soluciones, pero dos veces mejor que cuando no se da premedicación (C1) o se administra solo agua (C2) (Tabla 6).

Alas calificaciones dadas por los dos evaluadores de TVMS se aplicó la prueba $\chi^{2}$ con la que no hubo evidencia suficiente para pensar que los resultados son independientes; esto fue corroborado con la prueba de hipótesis de proporciones, en la cual se obtuvo un valor de $p=0,0000$ que permite rechazar la hipótesis nula que se planteó como inferior al $70 \%$. Al realizar el análisis para la variable categórica se encontró un acuerdo de 98 \% con el índice kappa de 0,8952, que indica una muy buena concordancia de interobservadores (Tabla 7 y Figura 4).

Respecto a la seguridad del estudio, no se presentó ningún evento adverso en los 412 pacientes en relación con intolerancia, hipersensibilidad, broncoespasmo, angioedema, exantema, prurito, hipotensión, náuseas, vómito, diarrea o alergias asociadas con el uso de SIM, NAC y HH. Tampoco hubo reacciones alérgicas, flebitis o depresión respiratoria con propofol y remifentanilo usados en la sedación. Es de resaltar que no se presentaron eventos de broncoaspiración en relación con la sedación consiente, teniendo en cuenta que, en 5 de las 6 cohortes, los pacientes ingirieron 100 $\mathrm{mL}$ de líquido entre 15 a 30 minutos previos al inicio de la endoscopia (media de 24,2 minutos). 
Tabla 6. Distribución en tabla cruzada de las cohortes (C1, C2, C3, C4, C5, C6) frente a la calidad de visibilidad TVMS excelente y adecuada

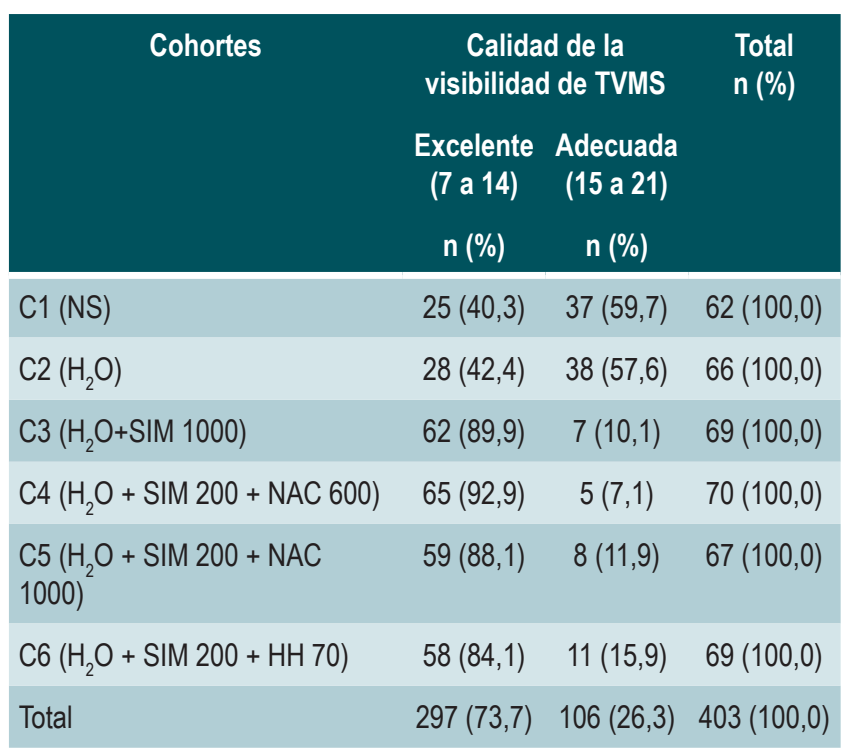

Tabla 7. Concordancia interobservadores en la calificación de visibilidad con TVMS propuesta

\begin{tabular}{|lccc}
$\begin{array}{l}\text { Calificación de la } \\
\text { calidad con TVMS }\end{array}$ & \multicolumn{2}{c}{$\begin{array}{c}\text { Concordancia interobservadores } \\
\text { (evaluadores) }\end{array}$} & $\begin{array}{c}\text { Total } \\
\mathbf{n}\end{array}$ \\
\cline { 2 - 3 } & Sí & No \\
\cline { 2 - 3 } & $\mathbf{n}(\%)$ & $\mathbf{n}(\%)$ & 296 \\
\hline Excelente (7 a 14) & $289(98)$ & $7(2)$ & 103 \\
\hline Adecuada (15 a 21) & $97(94)$ & $6(6)$ & 13 \\
\hline Inadecuada (22 a 28) & $8(62)$ & $5(38)$ & 12 \\
\hline Total & 394 & 18 & 412 \\
\hline
\end{tabular}

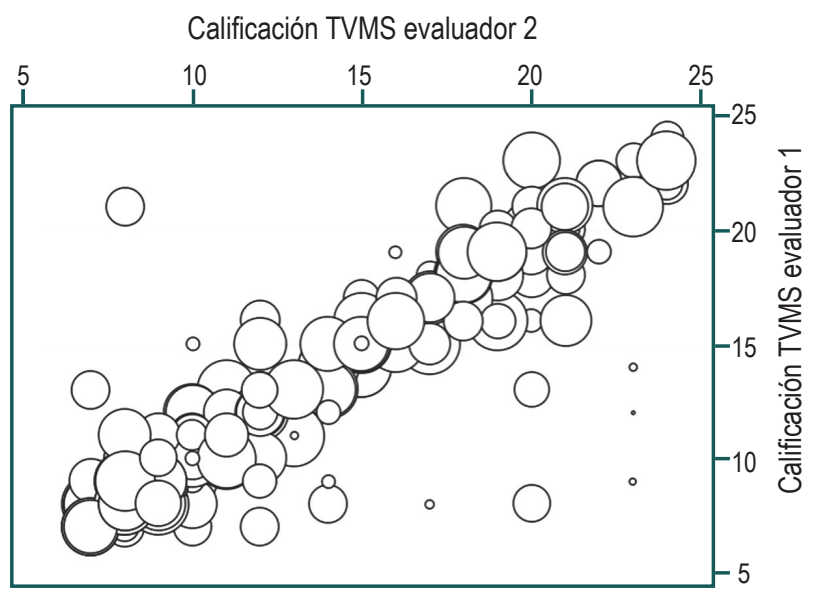

Figura 4. Concordancia de los interobservadores en la calificación de visibilidad con TVMS en una gráfica de burbujas.

\section{DISCUSIÓN}

El presente estudio de cohortes comparativas permitió confirmar que la mayor valoración de excelente visualización de la mucosa digestiva alta se asoció con el uso de premedicación con solución de NAC 600 mg + SIM 200 mg + agua $100 \mathrm{~mL}$, ingerida entre 15 y 30 minutos antes del paso del cricofaríngeo con el endoscopio. La premedicación con SIM con o sin NAC ha sido ampliamente estudiada con metaanálisis y revisiones sistemáticas. Lee, $\mathrm{Du}$ y Fu (12) incluyeron 5750 pacientes y la combinación de SIM con NAC mostró una mejor TVMS frente al uso de SIM sola; tanto SIM + NAC como SIM sola fueron más eficientes que el uso de agua sola (diferencias de medias $[\mathrm{MD}]=-0,14$ $[-0,25,-0,03] ; p=0,01)$, sin presencia de eventos adversos (usando SIM o dimeticona en dosis de 40 a $200 \mathrm{mg}$ y NAC entre 200 y hasta $1000 \mathrm{mg}$; comparado con grupos de control con agua entre $5 \mathrm{~mL}$ y $100 \mathrm{~mL}$ ). Todos reportaron TVMS, excepto uno que presentó una escala cualitativa de excelente, adecuado e inadecuado (6).

En otro metaanálisis, Sajid y colaboradores (11) incluyeron 654 pacientes, con o sin uso de SIM + / - NAC y encontraron una asociación de mejoría (Odds ratio [OR]: 0,43; IC $95 \%[0,28: 0,68] \mathrm{z}=3,65 ; p=0,003)$ en la visibilidad mucosa cuando se utilizó premedicación. Se analizó otro grupo de 364 pacientes y se encontró que el uso de SIM +/- NAC estuvo asociado con una mejoría en el puntaje de visibilidad mucosa comparado con el grupo en el que no se usó SIM (diferencia de media estandarizada [DME]: -1,66; IC $95 \%:-1,93,-1,40 ; z=12,12 ; p=0,00001)$. Aunque la calidad de visualización según la TVMS fue superior con las 4 cohortes de soluciones utilizadas frente al ayuno o al uso de agua $(88,7 \%$ frente a $41,4 \%)$, la mejor premedicación correspondió a la cohorte $\mathrm{C} 4\left(\mathrm{H}_{2} \mathrm{O}+\mathrm{SIM} 200+\mathrm{NAC}\right.$ 600 ), con un volumen de lavado de $59,9 \mathrm{~mL}$, inferior y significativamente diferente a las cohortes de no premedicación C1 y C2 $(p>0,001)$ y con un rendimiento de $92,9 \%$, superior a los grupos C3 $(89,9 \%)$, C5 $(88,1 \%)$ y C6 $(84,1$ $\%)$, aunque sin diferencia significativa entre ellas. Nuestra experiencia previa, aplicada en la cohorte $\mathrm{C} 3\left(\mathrm{H}_{2} \mathrm{O}+\mathrm{SIM}\right.$ $1000)$, mostró la segunda mejor eficiencia, con visibilidad excelente en $89,9 \%$, comparable con lo publicado por Bertoni (27) y Chang (30). Contrasta con otra publicación en la que se utilizó simeticona líquida a bajas dosis y volumen (SIM $1 \mathrm{~mL}$ de $100 \mathrm{mg}$, diluido a $5 \mathrm{~mL}$ de agua, administrado 30 minutos antes de la endoscopia), en la que reportan equivalencia de visión excelente en el 51,9\% (9), cifra apenas levemente superior a lo que encontramos en ayuno o con solo agua, razón por la cual no recomendamos ese esquema. El resultado de la cohorte $\mathrm{C} 5\left(\mathrm{H}_{2} \mathrm{O}\right.$ + SIM 200 + NAC 1000), que tuvo el tercer mejor rendimiento, mostró que la mezcla de NAC + SIM no fue una 
dosis dependiente del aumento de NAC y que, por tanto, su concentración de $600 \mathrm{mg}$ es suficiente e incluso mejor que usar $1000 \mathrm{mg}$ para lograr una visualización excelente. La cohorte C6 ( $\left(\mathrm{H}_{2} \mathrm{O}+\right.$ SIM $\left.200+\mathrm{HH} 70\right)$, con Hedera helix a baja y única dosis de $70 \mathrm{mg}$ (en uso fuera de indicación como alternativo a NAC) logró un porcentaje de excelencia de $84,1 \%$, superior a la no premedicación de C1 (40,3\%) y C2 (42,4\%); aunque su media de volumen de lavado $(83,4 \mathrm{~mL})$ no mostró una diferencia significativa frente a ellas. Deja así el campo abierto para futuras comprobaciones de su eficiencia, a otras concentraciones y en un estudio controlado, toda vez que puede ser una alternativa segura y costo-efectiva.

La endoscopia digestiva alta realizada en condición de solo ayuno de 6 a 8 horas tuvo una calidad de la visibilidad adecuada en solo el $25,7 \%$, es decir que 1 de cada 4 pacientes va a requerir por lo menos irrigación de 3 sitios de su tracto digestivo alto (con una media volumen de $125 \mathrm{~mL}$ ). En Chile, en las mismas condiciones, se requirió en promedio $52 \mathrm{~mL}$ de agua (rango entre 0 y $400 \mathrm{~mL}$ ) valorando solo 4 sitios, todos en el estómago (14). El uso de solo agua como premedicación (cohorte $\mathrm{C} 2\left[\mathrm{H}_{2} 0100 \mathrm{~mL}\right]$ ) no mostró una diferencia significativa frente al ayuno, con un volumen de limpieza levemente inferior al ayuno (media de 123 $\mathrm{mL}$ frente a $126 \mathrm{~mL}$ ). No confirmó otra descripción (6), en la cual el uso de $100 \mathrm{~mL}$ de agua empeoraba la TVMS, lo que había sugerido que el agua diseminaba el moco en un área más extensa del estómago, sin diluir moco o burbujas.

Frente al segundo objetivo del estudio, se logró aportar una nueva TVMS, escala adaptada y mejorada al tener en cuenta 7 sitios de valoración ( 1 del esófago, 4 del estómago, 1 del bulbo duodenal y 1 de la segunda porción del duodeno), extendiendo el concepto de la necesidad de excelente visibilidad más allá del estómago, que permitiera la detección de lesiones en la fase temprana diferentes al cáncer gástrico incipiente. La conversión cuantitativa a cualitativa de TVMS se realizó de forma sencilla: a cada uno de los 7 sitios se le asignaron puntajes de 1 a 4 , para una suma total entre 7 y 28 ; las calificaciones 1 y 2 , que significan que no había ningún residuo de burbujas, saliva o moco ( 1 de puntuación) o que, a lo sumo, estos residuos eran succionables ( 2 de puntuación). El rango de puntuación para una visibilidad excelente entonces estuvo entre 7 y 14, condición en la que solo se succionaba o la cantidad de irrigación fue mínima (menos de $50 \mathrm{~mL}$ ). Para la visibilidad adecuada, el rango se definió entre 15 y hasta 21 puntos, condición en la cual las burbujas, el moco o la saliva no eran completamente succionables y requerían irrigación de limpieza (en un volumen menor de $50 \mathrm{~mL}$, lo que cambia el puntaje de 2 a 3) en más de 4 sitios y hasta los 7 sitios. Se consideró visibilidad inadecuada cuando el rango estaba entre 22 (6 de los sitios requirieron volumen menor de $50 \mathrm{~mL}$, y 1 requirió más de $50 \mathrm{~mL}$ ) hasta 28 ( 7 sitios con lavado mayor de $50 \mathrm{~mL}$ ); estos puntajes recomendarían la interrupción del procedimiento y su reprogramación.

Entendida la escala, se confirmó su aplicabilidad y validez mediante el resultado de la concordancia interobservadores, con un acuerdo de $98 \%$ cuando la visibilidad según la TVMS fue excelente y de $94 \%$ cuando la visibilidad fue adecuada. La pretensión de la escala mixta (cualitativa y cuantitativa) fue facilitar la comparabilidad del efecto de las soluciones usadas como premedicación, con la particularidad que se construyó uniendo conceptos de la escala de Chang y colaboradores (30) en relación con el volumen de lavado de más o menos de $50 \mathrm{~mL}$; la escala de Lee y colaboradores (26), en cuanto a los 7 sitios de lavado, incluidos el esófago y el duodeno; la escala clásica de Kuo y colaboradores (28) y la de Asl y colaboradores (29), que usan grados de puntuación de 1 a 4, solo para los 4 sitios del estómago (con diferencia frente a nuestra escala, pues usaron un volumen de más o menos $30 \mathrm{~mL}$ ); y la escala de Elvas y colaboradores (7), que describió los tres grados cualitativos descritos. También se constituyó en una herramienta útil para cumplir el requerimiento reciente de informar la visualización de la mucosa como un parámetro de calidad intraprocedimiento (3).

Frente al tercer objetivo, no se presentaron eventos adversos o complicaciones relacionadas con el procedimiento de endoscopia; ni relacionados con los tres productos utilizados (SIM, NAC y HH) en las soluciones de premedicación de las cohortes C2 a C6. Tampoco hubo alergias o depresión respiratoria con propofol y remifentanilo utilizados en todos los pacientes; en relación con la sedación y coincidiendo con los estudios de Koeppe y colaboradores (32) y de Da Silva y colaboradores (33), en los cuales se permitió la ingesta de agua de $200 \mathrm{~mL}$ a $410 \mathrm{~mL}$ hasta 1 hora previa a endoscopia con solo una observación subjetiva de más líquido libre en el estómago, coincidimos en no haber presentado casos de broncoaspiración. De esta forma, los resultados mostraron que la premedicación bajo las condiciones de volumen, diluciones, tiempos, condiciones fisiológicas y bajo el esquema de sedación consciente utilizada (publicada por nosotros en 2017) (23) es una práctica segura y muy útil.

Una de las limitaciones del estudio es la participación de un solo gastroenterólogo, situación no solucionable dadas las condiciones propias de la institución, compensada con el entrenamiento y conocimiento preestudio tanto de la escala TVMS como las soluciones de premedicación) con 100 pacientes y la participación de todo el equipo de trabajo, incluida la enfermera coevaluadora. Para finalizar, la baja concentración de SIM utilizada en el líquido de limpieza está en relación con publicaciones que describen la presencia de sus residuos de SIM dentro del endoscopio, a 
pesar del reprocesamiento de alto nivel, que podría fomentar el crecimiento bacteriano $(34,35)$.

El estudio se adhirió a la Declaración de la Sociedad Gastroenterológica de Australia (2019), que ante la no evidencia "de eventos clínicos adversos durante décadas de su uso, nosotros creemos que el uso continuado de simeticona es apropiado y puede ser administrado a través de cualquier canal de endoscopia”. Y sus recomendaciones son las siguientes: el uso de SIM es razonable toda vez que mejora la visibilidad de la mucosa gástrica y colónica, y facilita la detección de adenomas en la colonoscopia (nivel de evidencia IA, grado de recomendación A); se debe usar la menor cantidad efectiva de simeticona para el fluido de lavado, de 2 a $3 \mathrm{~mL}$ de $120 \mathrm{mg} / \mathrm{mL}$ adicionados a un litro de agua estéril (nivel de evidencia IV, grado de recomendación D); SIM puede ser administrado oralmente o a través de cualquier canal de irrigación (nivel de evidencia IV, grado de recomendación D); y es esencial la estricta adherencia a los protocolos de reprocesamiento de los endoscopios, en especial la inmediata descontaminación prelimpieza al lado del paciente, que incluya irrigación posprocedimiento e inicio pronto de limpieza manual o mecánica (nivel de evidencia IIB, grado de recomendación B) (36).

\section{CONCLUSIONES}

Utilizar premedicación con SIM sola o mezclada con mucolíticos como NAC o HH (este último, recomendación de uso fuera de indicación) permite mejores tasas de visibilidad que cuando se realiza una endoscopia digestiva solo en ayunas o con solo administración de agua. De las 4 soluciones estudiadas, la de mejores resultados fue SIM 200 mg + NAC 600 $\mathrm{mg}+$ agua $100 \mathrm{~mL}$, con lo que se logró calidad de visibilidad excelente del 92,9\%; sin embargo, las otras 3 soluciones dan resultados excelentes por encima del $84,1 \%$. La escala TVMS propuesta, que incluye 7 sitios con 4 calificaciones y convertidas a 3 calificaciones cualitativas (excelente, adecuada e inadecuada) es una herramienta fácil de aplicar y más completa frente a escalas publicadas previamente. El esquema de premedicación de hasta $100 \mathrm{~mL}$, ingerida entre 15 y 30 minutos antes de la endoscopia, es una exposición segura en pacientes adultos ASA I y II, intervenidos bajo sedación consciente. El uso del mucolítico Hedera helix es una alternativa diferente y menos costosa, aunque menos eficiente que NAC.

\section{Agradecimientos}

El presente trabajo se realizó en el marco del Diplomado de Investigación Científica de la Universidad CES, en convenio y con financiación de la Asociación Colombiana de Gastroenterología, en la gestión y apoyo decidido del Dr. Fabio Gil Parada; al laboratorio Tecnoquímicas, por apoyar con el producto $\mathrm{N}$-acetilcisteína $\left(\right.$ Aflux $\left.^{\circledast}\right)$; al laboratorio Incobra S. A., por apoyar con el producto simeticonadimetilpolisiloxano (Siligás ${ }^{\circledR}$ ); y al laboratorio Roemmers, por apoyar con el producto Hedera Helix (Abrilar $\left.{ }^{\oplus}\right)$.

\section{REFERENCIAS}

1. Adams MA, Saini SD, Allen JI. Quality measures in gastrointestinal endoscopy: the current state. Curr Opin Gastroenterol. 2017;33(5):352-357. doi: 10.1097/ MOG.00000000000000379

2. Bisschops R, Areia M, Coron E, Dobru D, Kaskas B, Kuvaev R, Pech O, Ragunath K, Weusten B, Familiari P, Domagk D, Valori R, Kaminski MF, Spada C, Bretthauer M, Bennett C, Senore C, Dinis-Ribeiro M, Rutter MD. Performance measures for upper gastrointestinal endoscopy: A European Society of Gastrointestinal Endoscopy quality improvement initiative. United European Gastroenterol J. 2016;4(5):629-656. doi: $10.1177 / 2050640616664843$

3. Beg S, Ragunath K, Wyman A, Banks M, Trudgill N, Pritchard DM, Riley S, Anderson J, Griffiths H, Bhandari P, Kaye P, Veitch A. Quality standards in upper gastrointestinal endoscopy: a position statement of the British Society of Gastroenterology (BSG) and Association of Upper Gastrointestinal Surgeons of Great Britain and Ireland
(AUGIS). Gut. 2017;66(11):1886-1899. doi: 10.1136/ gutjnl-2017-314109

4. Koga M, Arakawa K. [On the application of enzymatic mucinolysis in $\mathrm{x}$-ray diagnosis of the stomach]. Nihon Igaku Hoshasen Gakkai Zasshi. 1964;24:1011-31.

5. Ida K, Okuda J, Nakazawa S, Yoshino J, Itoh M, Yokoyama $\mathrm{Y}$, et al. Clinical evaluation of premedication with KPD (Pronase) in gastroendoscopy-placebo-controlled double blind study in dye scatt ering endoscopy. Clin Rep. 1991;25:1793-804.

6. Monrroy H, Vargas JI, Glasinovic E, Candia R, Azúa E, Gálvez C, Rojas C, Cabrera N, Vidaurre J, Álvarez N, González J, Espino A, González R, Parra-Blanco A. Use of $\mathrm{N}$-acetylcysteine plus simethicone to improve mucosal visibility during upper GI endoscopy: a doubleblind, randomized controlled trial. Gastrointest Endosc. 2018;87(4):986-993. doi: 10.1016/j.gie.2017.10.005

7. Elvas L, Areia M, Brito D, Alves S, Saraiva S, Cadime AT. Premedication with simethicone and $\mathrm{N}$-acetylcysteine in improving visibility during upper endoscopy: a double- 
blind randomized trial. Endoscopy. 2017;49(2):139-145. doi: $10.1055 /$ s-0042-119034

8. Callaghan JL, Neale JR, Boger PC, Sampson AP, Patel P. Variation in preparation for gastroscopy: lessons towards safer and better outcomes. Frontline Gastroenterol. 2016;7(3):187-190. doi: 10.1136/flgastro-2015-100647

9. Song M, Kwek AB, Law NM, Ong JP, Tan JY, Harichander Thurairajah P, Ang DS, Ang TL. Efficacy of small-volume simethicone given at least $30 \mathrm{~min}$ before gastroscopy. World J Gastrointest Pharmacol Ther. 2016;7(4):572-578. doi: 10.4292/wjgpt.v7.i4.572

10. Bertoni G, Gumina C, Conigliaro R, Ricci E, Staffetti J, Mortilla MG, Pacchione D. Randomized placebo-controlled trial of oral liquid simethicone prior to upper gastrointestinal endoscopy. Endoscopy. 1992;24(4):268-70. doi: $10.1055 / \mathrm{s}-2007-1010479$

11. Sajid MS, Rehman S, Chedgy F, Singh KK. Improving the mucosal visualization at gastroscopy: a systematic review and meta-analysis of randomized, controlled trials reporting the role of Simethicone $\pm \mathrm{N}$-acetylcysteine. Transl Gastroenterol Hepatol. 2018;3:29. doi: 10.21037/ $\operatorname{tgh} .2018 .05 .02$

12. Li Y, Du F, Fu D. The effect of using simethicone with or without $\mathrm{N}$-acetylcysteine before gastroscopy: A metaanalysis and systemic review. Saudi J Gastroenterol. 2019 Jul-;25(4):218-228. doi: 10.4103/sjg.SJG_538_18

13. Zhang LY, Li WY, Ji M, Liu FK, Chen GY, Wu SS, Hao Q, Zhai HH, Zhang ST. Efficacy and safety of using premedication with simethicone/Pronase during upper gastrointestinal endoscopy examination with sedation: A single center, prospective, single blinded, randomized controlled trial. Dig Endosc. 2018;30(1):57-64. doi: 10.1111/den.12952

14. Mansilla R, Uslar T, Chahuán J, Latorre G, Cruz R, Cruz R, Sirhan M, Espino A, Honold F, Huenur J, Miranda P, Riquelme A. Validez y confiabilidad de una escala de clasificación de limpieza gástrica en endoscopia digestiva. Gastroenterol Latinoam. 2016;27(1): 9-17.

15. Chang WK, Yeh MK, Hsu HC, Chen HW, Hu MK. Efficacy of simethicone and $\mathrm{N}$-acetylcysteine as premedication in improving visibility during upper endoscopy. J Gastroenterol Hepatol. 2014;29(4):769-74. doi: 10.1111/ jgh. 12487

16. Buxbaum JL, Hormozdi D, Dinis-Ribeiro M, Lane C, Dias-Silva D, Sahakian A, Jayaram P, Pimentel-Nunes P, Shue D, Pepper M, Cho D, Laine L. Narrow-band imaging versus white light versus mapping biopsy for gastric intestinal metaplasia: a prospective blinded trial. Gastrointest Endosc. 2017 Nov;86(5):857-865. doi: 10.1016/j. gie.2017.03.1528

17. World Source: Globocan 2020 [Internet]. IARC; 2020 [citado el 5 de febrero de 2020]. Disponible en: https:// gco.iarc.fr/today/data/factsheets/populations/900-worldfact-sheets.pdf

18. Wiesner Ceballos C, Henríquez Mendoza GM, Aguilera López J. Análisis de situación del cáncer en Colombia: 2015. Colombia, Ministerio de Salud, Instituto Nacional de
Cancerología; 2017. Disponible en: https://www.cancer. gov.co/Situacion_del_Cancer_en_Colombia_2015.pdf

19. Matsuzaka M, Fukuda S, Takahashi I, Shimaya S, Oyama T, Yaegaki M, Shimoyama T, Sakamoto J, Nakaji S, Umeda $\mathrm{T}$. The decreasing burden of gastric cancer in Japan. Tohoku J Exp Med. 2007;212(3):207-19. doi: 10.1620/ tjem.212.207

20. Kim GH, Liang PS, Bang SJ, Hwang JH. Screening and surveillance for gastric cancer in the United States: Is it needed? Gastrointest Endosc. 2016;84(1):18-28. doi: 10.1016/j.gie.2016.02.028

21. Gómez-Zuleta MA, Ruíz-Morales OF, Otero-Regino W. Efectividad de la premedicación con $\mathrm{N}$-acetil cisteína más dimetilpolisiloxano versus un placebo para mejorar la visibilidad en la endoscopia digestiva: estudio prospectivo, ciego, controlado aleatorizado. Medicina (Mex). 2017;39(2):98-106.

22. Royero Gutiérrez HA. Aplicación de una escala de visualización de la mucosa gástrica, durante la esofagogastroduodenoscopia en pacientes premedicados con N-acetilcisteína más simeticona: experiencia en Ocaña, Norte de Santander. Rev Colomb Gastroenterol. 2018;33(1):1-7. https://doi. org/10.22516/25007440.226

23. Blanco Avellaneda CJ, García KR, Molano A, Chimbi DY, Forero AY. Seguridad y eficiencia de sedación balanceada con propofol y remifentanil en endoscopia digestiva alta diagnóstica. Una experiencia exitosa. Rev Colomb Gastroenterol. 2017;32(2):120-30. https://doi. org/10.22516/25007440.140

24. McNally PR, Maydonovitch CL, Wong RK. The effectiveness of simethicone in improving visibility during colonoscopy: a double-blind randomized study. Gastrointest Endosc. 1988;34(3):255-8. doi: 10.1016/s00165107(88)71324-3

25. Bhandari P, Green S, Hamanaka H, Nakajima T, Matsuda T, Saito Y, Oda I, Gotoda T. Use of Gascon and Pronase either as a pre-endoscopic drink or as targeted endoscopic flushes to improve visibility during gastroscopy: a prospective, randomized, controlled, blinded trial. Scand J Gastroenterol. 2010;45(3):357-61. doi: 10.3109/00365520903483643

26. Lee GJ, Park SJ, Kim SJ, Kim HH, Park MI, Moon W. Effectiveness of Premedication with Pronase for Visualization of the Mucosa during Endoscopy: A Randomized, Controlled Trial. Clin Endosc. 2012;45(2):161-4. doi: 10.5946/ce.2012.45.2.161

27. Bertoni G, Gumina C, Conigliaro R, Ricci E, Staffetti J, Mortilla MG, Pacchione D. Randomized placebo-controlled trial of oral liquid simethicone prior to upper gastrointestinal endoscopy. Endoscopy. 1992;24(4):268-70. doi: $10.1055 / \mathrm{s}-2007-1010479$

28. Kuo CH, Sheu BS, Kao AW, Wu CH, Chuang CH. A defoaming agent should be used with pronase premedication to improve visibility in upper gastrointestinal endoscopy. Endoscopy. 2002;34(7):531-4. doi: 10.1055/s-200233220 
29. Asl SM, Sivandzadeh GR. Efficacy of premedication with activated Dimethicone or $\mathrm{N}$-acetylcysteine in improving visibility during upper endoscopy. World J Gastroenterol. 2011;17(37):4213-7. doi: 10.3748/wjg.v17.i37.4213

30. Chang CC, Chen SH, Lin CP, Hsieh CR, Lou HY, Suk FM, Pan S, Wu MS, Chen JN, Chen YF. Premedication with pronase or $\mathrm{N}$-acetylcysteine improves visibility during gastroendoscopy: an endoscopist-blinded, prospective, randomized study. World J Gastroenterol. 2007;13(3):444-7. doi: 10.3748/wjg.v13.i3.444

31. Pimentel-Nunes P, Libânio D, Marcos-Pinto R, Areia M, Leja M, Esposito G, Garrido M, Kikuste I, Megraud F, Matysiak-Budnik T, Annibale B, Dumonceau JM, Barros R, Fléjou JF, Carneiro F, van Hooft JE, Kuipers EJ, DinisRibeiro M. Management of epithelial precancerous conditions and lesions in the stomach (MAPS II): European Society of Gastrointestinal Endoscopy (ESGE), European Helicobacter and Microbiota Study Group (EHMSG), European Society of Pathology (ESP), and Sociedade Portuguesa de Endoscopia Digestiva (SPED) guideline update 2019. Endoscopy. 2019;51(4):365-388. doi: 10.1055/a-0859-1883

32. Koeppe AT, Lubini M, Bonadeo NM, Moraes I Jr, Fornari F. Comfort, safety and quality of upper gastrointestinal endoscopy after 2 hours fasting: a randomized controlled trial. BMC Gastroenterol. 2013;13:158. doi: 10.1186/1471-230X-13-158
33. De Silva AP, Amarasiri L, Liyanage MN, Kottachchi D, Dassanayake AS, de Silva HJ. One-hour fast for water and six-hour fast for solids prior to endoscopy provides good endoscopic vision and results in minimum patient discomfort. J Gastroenterol Hepatol. 2009;24(6):1095-7. doi: 10.1111/j.1440-1746.2009.05782.x

34. Ofstead CL, Wetzler HP, Johnson EA, Heymann OL, Maust TJ, Shaw MJ. Simethicone residue remains inside gastrointestinal endoscopes despite reprocessing. Am J Infect Control. 2016 Nov 1;44(11):1237-1240. doi: 10.1016/j.ajic.2016.05.016

35. Sutton S, Jimenez L. A Review of Reported Recalls Involving Microbiological Control 2004-2011 with Emphasis on FDA Considerations of "Objectionable Organisms" [Internet]. American Pharmaceutical Review; 2012 [citado el 21 de febrero de 2020]. Disponible en: http://www.americanpharmaceuticalreview.com/ Featured-Articles/38382-A-Review-of-Reported-RecallsInvolving-Microbiological-Control-2004-2011-withEmphasis-on-FDA-Considerations-of-ObjectionableOrganisms/

36. Devereaux BM, Taylor ACF, Athan E, Wallis DJ, Brown RR, Greig SM, Bailey FK, Vickery K, Wardle E, Jones DM. Simethicone use during gastrointestinal endoscopy: Position statement of the Gastroenterological Society of Australia. J Gastroenterol Hepatol. 2019;34(12):20862089. doi: $10.1111 /$ jgh.14757 